\title{
مجلـةبحــوث
}

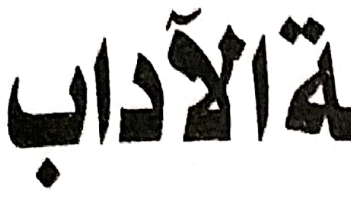

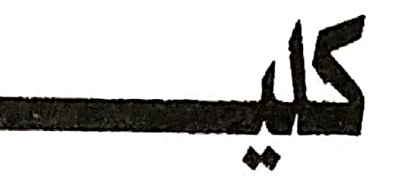

\section{البحث) (IN)}

L'espoir de la justice est l'ambition des opprimés dans : "L'élephant ô roi du temps " pièce de Saadallah Wannous.

\section{By}

Dr. Iman Mahmoud Zahran

Maitre de Conférence - Université de Minia

Faculté des Lettres

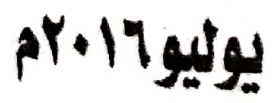 \\ العلد (1.7) (1.7) \\ rraiul|}

http : // Art.menofia . edu. eg *** E- mail: rifa2012@ Gmail.com 
L'espoir de la justice est l'ambition des opprimés dans

$L$ 'espoir de la justice est l'ambition des opprimés dans : «L'éléphant ô roi du temps » pièce de Saadallah Wannous.

\section{Iman Mahmoud Zahran \\ Maître de Conférences \\ Université de Minia \\ Faculté des Lettres \\ Introduction}

« Pour accéder à la liberté, il faut n'être ni maître ni esclave. Hegel ${ }^{1}$

«Ceux qui acceptent de se soumettre sont donc dénaturés, ils ne sont plus alors des hommes. ${ }^{2}$ "

La relation entre le gouvernant et le gouverné est une problématique politique qui a occupé l'esprit de nombreux écrivains arabes.

Wannous est un grand dramaturge syrien considéré comme l'un des rares écrivains arabes les plus importants qui ont consacré leur production dramatique à cette question élaboré à l'aide d'une technique professionnelle avec: «L'Éléphant, ô roi du temps. \& traite la relation contradictoire : l'oppression par le gouverneur en retour, de peur d'être pré-condamné, Ici le peuple servile fait d'un gouverneur, un tyran. "Les peuples sont responsables de leur mise sous tutelle $\iota^{3}$

Saadallah Wannous, est un dramaturge syrien, décédé en 1997 à l'âge de 56 ans. Le théâtre de Saadallah Wannous est un théâtre de l'humanité. Il met en scène, la torture des peuples arabes et les crimes des rois, et dans quelle mesure ces crimes malheureusement sont devenus une habitude pour ces peuples opprimés. "Ses pièces ont marqué le théâtre syrien et arabe, et sont traduites dans beaucoup de langues. Les plus célèbres

1 G.W.F. Hegel, Principes de la philosophie du droit, texte présenté, traduit et annoté par R.

Derathé, Vrin, Paris, 1975, p. 296.

2 Gouin, Rodolphe (2007), « Servitude volontaire », in V. Bourdeau et R. Merrill (dir.),DicoPo,

3 Ibid.

V9V 


\section{Dr. Iman Mahmoud Zahran}

sont : Fête pour le 5 juin, L'aventure de la Tête du mamelouk Jâbir, Le roi est le roi, et L'éléphant ô roi du temps. " ${ }^{4}$

Wannous a commencé à écrire de courts récits. Après plusieurs tentatives, il décide d'écrire pour le théâtre. Il justifie ce choix dans une interview accordée à Sulaymān al-Hakim dans la revue 'Adab wa naqd:

Il m'est arrivé une fois de dire que j'ai choisi le théatre parce que je sentais en moi un penchant vers la dialectique, mais j'ai pris conscience que mon choix pour le théâtre découlait de ma tendance intellectuelle pour l'abstraction et non pour la narration. Cependant, j'ai découvert ultérieurement que ma motivation profonde pour le théâtre venait d'un désir de réaliser une ouvre d'une portée actuelle. ${ }^{5}$ "

\section{Wannous est conscient que :}

"Le théâtre est né politique, et il l'est reste. ${ }^{6}$ "

On peut souligner que Wannous s'intéresse dans cette période, les rapports de l'homme avec la société, car pour lui : «l'homme a sur la scène l'importance d'une fonction sociale ${ }^{7} 》$.

On a préféré réaliser notre étude sur une des pièces qui date entre 1967 et 1978, où, on enregistre une écriture ambitieuse dont le théâtre de la politisation est l'idée principale de ces pièces. Il semble que les sentiments de la réfraction après la défaite arabe de 1967 dominent les drames de Wannous de cette époque et avèrent : « un sentiment de perte personnelle et collective ${ }^{8} »$

L'Éléphant, Ô roi du temps !, n'est pas le titre d'un film de dessin animé pour le plaisir des enfants, mais en effet elle est le titre de la pièce simule une réalité amère. Cette pièce de

4 Mohammed Habib Samrakandi, Le théâtre arabe au miroir de lui-même et son contact avec les créations des deux rives de la Méditerranée Numéro 58 de Horizons maghrébins P:150

5 Tomiche, Nada, La littérature arabe contemporaine roman, nouvelle, théâtre. Maisonneuve Edi. Larousse, Paris, 1981, p .131.

6 Wannous. Saadallah. (Euvres complètes, Al-a'mal al-kāmila (Euvres complètes), , Damas, al- Ahăhĭ, 1996, tome 3, p. 35.

7 Cette formulation est empruntée à Erwin Piscator, Le théâtre politique, p. 128.

8Le théâtre arabe au miroir de lui-même et son contact avec les créations des deux rives de la Méditerranée Presse Univ du Mirail, 2008 P : 150. 
L'espoir de la justice est l'ambition des opprimés dans

Tecrivain Saadallah Wannous reflète la triste réalité d'une société qui accepte l'injustice et l'humiliation et n'ose même pas se plaindre. Le génie dramatique de Wannous est distingué dans cette pièce Al-fil yā malik al-zamān (L'éléphant. Ô roi du temps !), par l'aspect et les durées brèves. Malgré que les pages de cette pièce ne dépassent pas 24 pages mais elle est riche d'evènements, il nous présente ses personnages dans des espaces différents. Ces pages sont concentrées en une scène partagée en quatre tableaux, dont chacun s'intitulé :

1. La décision.

2. Entraînements.

3. Devant le palais du roi.

4. Face au roi : « Wannous qui se considère comme étant un des pionniers du théâtre arabe contemporain, a commencé son projet d'écriture dramatique avec une série de pièces brèves. ${ }^{9}$ "

«Cette pièce a été présentée pour la première fois en 1969 au premier festival de théâtre de Damas créé à l'initiative de Saadallah Wannous.

Le soir de la première en 1971 , la représentation dont la mise en scène était de Wannous fut interdît $(. ..) n^{10}$

La pièce raconte l'histoire du roi injuste qui possède un éléphant qui a causé de graves dommages à la paroisse, personne n'ose se plaindre. Le grand désastre lorsque l'éléphant écrase un enfant sous ses pieds dans une des ruelles. Le groupe rétréci. Dans ce moment apparaît sur la scène Zakariyya héros populaire incite à envisager le roi et à la révolte. Il encourage la paroisse à la plainte et il la convainc que tout le monde doit partager le fardeau de cette plainte dont Zakariyya commence criant: L'Éléphant, ô roi du temps, et la paroisse répond : nous ne voulons pas et elle commence à énumérer ses griefs. Le moment décisif lorsqu'elle se met debout devant le roi leur position a

9 Ibid.

10 Tomiche, Nada, La littérature arabe contemporaine roman, nouvelle, théâtre. Maisonneuve Edi.

Larousse, Paris, 1981, p. 131. 
changé complètement et le groupe se tait et la crainte le domine à nouveau et Zakariyya répétant: L'Éléphant, ô roi du temps, et le roi lui demande que? Personne ne répond à la question devant le roi, avec l'escalade de la tonalité de sa voix menacée, Zakariyya se trouve tout seul, semblait répondre à la question en disant au roi : " Nous exigeons marier l'éléphant et nous a des dizaines d'éphants ... éléphants ... des centaines de milliers d'éléphants. »

Le théâtre de Wannous est bien influencé par les écritures occidentales où il s'assure avec les drames de formules patriotiques : «Cette pièce confirme encore une fois la mise en pratique et l'innovation des idées brechtiennes du théâtre épique chez Wannous. Wannous fait appel à un conte populaire inspiré de la tradition orale, et il discute, à travers ce conte ancien, un sujet politique actuel ${ }^{11}$. $)$ Les thèmes de cette pièce sont variés. Notre étude a pour but d'expliquer dans la première partie comment la pièce de Wannous est « un violent réquisitoire contre la tyrannie ${ }^{12}$

Dans sa pièce il pose une question essentielle : "pourquoi obéiton? ». Selon lui, le roi ne peut enchaîner les gens s'ils ne s'enchaînent pas d'abord eux-mêmes. L'homme devient libre s'il ne veut plus servir : "Soyez résolus à ne plus servir, et vous voilà libres ${ }^{13}$ Ainsi Wannous tente de comprendre pourquoi le peuple a abandonné l'ambition de regagner sa liberté perdue. La pièce a pour but d'expliquer cette sujétion, afin de pousser les hommes à lutter pour s'en libérer. Dans cette partie, nous examinerons la relation que Wannous établit entre le roi et son peuple.

Dans la deuxième partie de ce travail, nous allons illustrer la théorie de la servitude volontaire de Etienne La Boétie, Wannous présente les éléments principaux de cette théorie dans cette pièce où il prône le théâtre politique. Avec cette 
L'espoir de la justice est l'ambition des opprimés dans

auvre Wannous conduit to thétre de langue arabe à une station importante.

Lierasement de peuple face au pouvoir de l'éléphant:

Il nous semble important de préciser que l'écrivain a choisi un

titre L'Éléphant, ô rol du temps comme une phrase incomplete, ce qui rend clair, qu'il est possible de trouver après cette phrase des injustices de l'éléphant fortement et hardiment. Parce que la peur déboucher leurs bouches, par ce que le peuple éablit a l'intérieur un mur de la peur, il mérite cette oppression,

et Wannous affirme que la révolution n'a pas réussi dans les âmes que la peur a brisé. L'accusation devient violente avec ce reproche qui montre que "c'est le peuple qui a bâti le tyran. ${ }^{14}$ "

En effet les évènements de cette pièce se déroulent entre deux espaces, le premier est rural dans le but de présenter les misères du peuple et les injustices qu'il endure, le second espace est le palais qui reflète leur anxiété et leur peur devant la vie luxueuse de roi.

En effet, Wannous dénonce la question du pouvoir comme nous allons la confirmer par la suite. Cette pièce de Wannous s'est inspirée d'une histoire populaire où il nous expose la frayeur des hommes d'un éléphant gâté par le roi. L'unique but du peuple est que le roi devait être jugé en simple citoyen ; mais en effet le roi est jugé en ennemi .Le premier tableau s'appelé : la décision où Wannous décrit le malheur qui aiguise la colère des gens est la mort d'un petit garçon écrasé par l'éléphant du roi :

"L'homme 1: Et ça s'est passé où?

L'homme 2 : Près de la boutique d'Ahmad Izzat. Là-bas la rue n'est jamais vide d'enfants ${ }^{15}$ '”

Cet accident a causé la mort d'un enfant :

14 lbid.

15 L'éléphant Ó roi du Temps! Pièce de Saadallah Wannous écrit en 1969, traduit en français par Batoul Jalabi-Wellnitz, Publications de l'Institut français du Proche-Orient

Chapitre I. al-fī yā Malik Al-Zamān, 2006. p. 453. 
"Les enfants jouaient dans la rue lorsque l'éléphant les surprit de rien. Les enfants prirent peur et ils s'enfitirent en courant. A part le fils de Muhammad al-Fahd, qui trébucha et s'étala par
terre.

Une telle terreur l'avait saisi qu'il ne pouvait plus se relever, l'éléphant l'atteignit et l'écrasa. ${ }^{16} \gg$

Ce tableau est composé spécialement de scène de foule où la population, s'unit dans le but de dénoncer les dommages que l'éléphant du roi a causé : les crimes de roi et son éléphant sont partout écrits avec le sang du peuple, il détruit les domiciles, aplatit le troupeau, détruit les plantes. La mort du petit enfant a fait soulever la colère du peuple, celui-ci est incapable de faire face à cette catastrophe. Petit à petit, la scène se remplit des différents personnages qui entrent de tous les côtés de la scène :

"Tous ceux qui entrent (en scène) se rejoignent les uns les autres et se rassemblent dans la rue. ${ }^{17}$ »

Par ce que les gens se gémissent contre l'acte destructeur de l'éléphant du roi. Il faut noter que ce tableau montre un manque de communication, personne ne les écoute vraiment. En effet ils cherchent à faire un dialogue, mais ils ne sont pas capables de se comprendre ou de faire un discours un peu sérieux. Un homme fournit des indications touchant la tragédie de la victime :

«L'homme 3 : Il lui a écrasé la poitrine et même le bas de la poitrine.

J'ai vu de mes propres yeux comment son ventre a éclaté et ses tripes se sont mélangées à la poussière de la rue. ${ }^{18}$ " 
L'espoir de la justice est l'ambition des opprimés dans

\section{peuple Indigent et malheureux :}

Parce qu'à la base, le roi emploie plusieurs astuces pour affaiblir

et écraser le peuple ses grands buts, sont : la pauvreté, La maladie et l'ignorance. Selon lui: «Enlever sous vos yeux le plus beau et le plus clair de votre revenu ${ }^{19}$ " le roi a connu profondément l'art de diviser les hommes il s'efforça de

paralyser une puissance. Ainsi s'explique que, le peuple est victime de la violence du roi. Comme le montre son éléphant qui menace la vie même de son peuple, et ce de deux manières. $\mathrm{Au}$ début il y a une violence quotidienne, le but du roi est de pouvoir exercer sur les sujets tous les moyens de contraintes possibles afin de protéger au mieux ses intérêts, l'éléphant est devenu un moyen de pression pour obtenir ce résultat. Puisque le peuple doit être maintenu dans son esclavage : « piller et dévaste vos champs...... tant de mains pour vous frapper, vous assaillir ${ }^{20}$.Ensuite, il faut servir les ambitions et les convoitises du roi. Tous ces dégâts et ces malheurs sont limités dans cette phrase négative :

«Vous vivez de telle sorte que rien n'est plus à vous »" ${ }^{21}$

Le tableau premier est un tableau tragique, lorsque l'éléphant tue le petit. En effet le peuple a une totale soumission par rapport à la figure de l'éléphant. Le peuple passe sa vie à obéir le roi qui peut vraiment avoir le contrôle sur leurs pensées, le roi se sert du peuple pour ses intérêts, comme s'il était un objet, sans âme et sans sentiments. En effet à certains moments, il veut se rebeller et protester contre son mauvais roi, dans d'autres moments il montre toute sa soumission et il cherche à gagner la sympathie de roi. On comprend qu'il ne sait pas quoi faire. On retrouve donc toujours le lien entre ces deux termes : l'obéissance et la protestation. Comment le peuple a-t-il été tranquille au milieu des périls qui le pressaient de toutes parts?

19 Thomas More, Livre 1, Aimer-la-littérature La Boétie, Discours de la servitude volontaire, 1576 Posté dans Essai, La Renaissance par cotentinghislaine

20 Ibid.

21 lbid. 
L'autoritarisme est une cruauté exercée contre le peuple qui

endure sans pouvoir protester. Wannous montre que le peuple se retrouve victime des pratiques corrompues et dommageables de l'éléphant :

"La femme 1: Les enfants sont écrasés dans les rues, La femme 3 : Et personne n'ose prononcer une parole. Les deux hommes 3 et 5 : Une parole !

La femme 2: Pas de sécurité pour les biens et les vies.

La femme 3 : Et personne n'ose prononcer une parole.

Les deux hommes 3 et 5 : Une parole $!^{22} »$

D'autre part, Parce que l'éducation et la connaissance donnent le pouvoir, la ruse du roi est de priver son peuple de ces droits, il s'efforce toujours de rendre les gens plus faibles et plus lâches :

«La liberté serait entièrement perdue et bannie de ce monde ${ }^{23}$ "

Wannous dégage cette pensée sociale exposée dans la pièce :

Ce système, cette pratique, ces allèchements étaient les moyens qu'employait le roi pour endormir leurs sujets dans la servitude. Mais ces misérables, se contentent d'endurer le mal. La pauvreté, la maladie et l'ignorance sont les maux majeurs que confronte la paroisse devenant les principaux moyens utilisés pour : « endormir les sujets dans la servitude ${ }^{24} \gg$ les sujets plongent dans ces problèmes majeurs qui les font perdre leurs identités et leurs hardiesses nécessaires au combat pour sortir de la servitude.

Ce moyen ne permet de dominer que les pauvres et les ignorants. Wannous a vivement rejeté le peuple malvoyant celui-ci a besoin d' un héros pour le guider à retrouver son intérêt. Puisque

23 Thomas More, Livre 1,Aimer-la-littérature La Boétie, Discours de la servitude volontaire, 1576 Posté dans Essai, La Renaissance par cotentinghislaine

$24 \mathrm{La}$ Boétie, Le discours de la servitude volontaire, Paris : Payot. November 2002 
L'espoir de la justice est l'ambition des opprimés dans

celui-ci est " aveugle à son bien » sa première provocation est, en effet, "de l'amener à ouvrir les yeux », d'abandonner l'ignorance et d'être : " un peuple instruit et pourra réfléchir à sa situation. ${ }^{25}$,

Ces traits se trouvent chez un personnage appelé Zakariyya. Il est une figure exemplaire, un type de héros réel. Comme nous allons voir dans la pièce, il se distingue des autres personnages par sa personnalité et de ses actes.

La vie du héros populaire est une vie normale, mais se modifie par des incidents graves. Il est le défenseur du peuple contre l'injustice sociale et l'oppression du pouvoir. Zakariyya incarne justement le héros populaire qui serait le dernier abri et le miroir de son peuple.

Il entre en scène et range une chorale dont il s'annonce le dirigeant. Les gens s'expriment d'une seule voix. Il est évident que, Zakariyya, un homme habile essaie modifier le comportement d'autrui. Son rôle est de convaincre : « le peuple doit arrêter de se croire inférieur à son gouvernement ${ }^{26}{ }_{\|}$

Le jeune héros Zakariyya traduit: « une vision optimiste de la chute possible du tyran par le refus de se soumettre à lui ${ }^{27}$.»

Mais pour appliquer cette vision optimiste, ce peuple doit posséder la décision de changer sa condition. L'obtention de la liberté est soulignée par cette idée qui prend la forme impérative « soyez résolus de ne servir plus, et vous voilà libres », et cette détermination consolide leur croyance et la libération apparaît réalisable. Zakariyya les pousse à avancer courageusement à leur but.

Il faut bien indiquer que ce n'est pas au roi mais au peuple que s'attache Wannous. En posant une question sur la relation mutuelle entre le dominé et le dominant. Telle est la leçon,

25 Ibid.

26 Lionel Hottin, La Boétie, Discours de la servitude volontaire, analyse, 3 août 2012 http_WWW.dokamo.nc-la-Boétie-de-la-servitude-volontaire-analyse

27 Thomas More, Livre 1, Essai 29 Déc.2012, Etienne de La Boétie, « Discours de la servitude volontaire ", 1576 - Corpus : Le modèle social humaniste à la Renaissance 


\section{Wannous souligne que le roi se montre agissant de détacher tout courage à son peuple.}

Il est certain que, la liberté de l'homme se retourne contre ellemême, lorsqu'il perd sa volonté, il devient la victime de la servitude. En outre, la pièce de Wannous affirme que le roi ne peut utiliser cette force de domination que par le consentement des sujets. Le peuple est coupable de sa : " dépossession volontaire de la liberté, » Le point central de la domination est ainsi le refus de s'assumer la liberté.

"Pour La Boétie, la liberté n'est pas l'objet de la volonté, mais désir (volonté) et liberté sont confondus : désirez et vous êtes libre, car un désir qui n'est pas libre n'est pas concevable, n'est pas un désir. » La liberté c'est ce que nous sommes, et si vous n'êtes pas libre, c'est que vous avez renoncé à votre désir. Dans le deuxième tableau, «Tadrïbät » (Entraînements), il y a tous les personnages du premier tableau :

«La scène est allumée. Une place publique. Les gens sont rassemblés, Zakariyyā se tient devant eux. ${ }^{28} \gg$.

L'arrivée de Zakariyyā - lui et l'enfant Mohamed décédé sont les seuls personnages nommés dans la pièce forme une transmutation dans l'action. Au début, Zakariyya s'annonce le responsable d'union et ordonne aux habitants de se joindre pour aller protester contre le roi :

«Zakariyyā : Moi, je vous dis ce que nous pouvons faire. Nous y allons tous ensemble et nous nous plaignons auprès du roi. ». Tout de suite un homme répondant en peur : «Qui sommes-nous pour entrer le palais du roi $^{29} "$ Les uns semblent craindre de porter la peine du courage ; ils sont marqués de l'impuissance de l'esprit humain .Par ce qu'il est inutile de demander à une foule ignorante de réfléchir, Zakariyya voit qu'il vaut mieux utiliser le langage des émotions. 
L'espoir de la justice est l'ambition des opprimés dans

Les événements sont inquiétants, les gens ne sont capables de bien vivre, en même temps, ils n'ont pas le courage de briser cette soumission. Zakariyya est pour eux ce personnage mystérieux, le personnage le plus important, il signifie le symbole de la liberté .C'est lui la voix de la colère qui montre aux sujets qu'il faut d'abord éliminer la pensée négative et la peur pour arriver à la voie de la victoire. L'œuvre de Wannous représente sans doute une révolution théâtrale, l'élément le plus révolutionnaire est représenté par les personnages, les hommes aveuglés caractérisés par une idée fixe de vivre en marginaux, n'ont pas d'identité sociale. Ils ne font rien pour rendre leur condition meilleur. Donc on souligne l'aspect dramatique de la vie humaine lorsque les personnages ne présentent pas une classe sociale spécifique, car ils ne font rien pendant qu'ils endurent les crimes de l'éléphant de roi. Comme nous allons voir Zakariyya se présente l'éveilleur de cette action de protestation. L'unique apte de conduire les gens. Si le peuple fait une révolte, si le peuple ose confronter le roi et ne l'obéit pas, la désobéissance suffit à détruire les jougs de la servitude, parce que les rois comme l'affirmation de La Boétie: « Ne sont grands que parce que nous sommes à genoux ${ }^{30}$ »

En effet, Zakariyya débute à arranger le groupe à faire face au souverain. Puis il les entraîne de parler ensemble afin que la complainte soit décisive. Ce modèle de protester montre que la responsabilité n'est pas individuelle mais collective, la représentation commune de leur plainte indique une allusion sérieuse :

«Zakariyyā : Comme je vous l'ai dit maintes fois. L'important c'est l'ordre, que nous n'ayons qu'un seul mot et une seule voix. Plus nos voix sont unies, plus leur impact est grand. ${ }^{31}$ "

Donc, Zakariyya essaie d'ordonner une « chorale » qui va répéter face au roi les terreurs de son éléphant, puis Zakariyyā,

30 La Boétie.

31 L'éléphant Ô roi du temps, p. 466 
prononce le préambule de la protestation devant le roi disant: "Al-fíl yā malik al-zamān» (L'éléphant! Ô roi du temps!) Pourvu que leur gémissement soit général le groupe parle de la même voix:

«Zakariyyä : [...] Allez... L'éléphant! Ô roi du temps! Le groupe : (Les voix commencent à s'harmoniser) Il a tué le fils de Muhammad al-Fahd [...]

Zakariyā : L'éléphant! Ô roi du temps !

Le groupe: Il y a quelques jours, il a failli tuer Abü Muhammad Hassāan [...]

Zakariyyä : L'éléphant ! Ô roi du temps !

Le groupe : Il a cassé les palmiers [...]

Zakariyyā : L'éléphant ! Ô roi du temps!

Le groupe : Il a démoli la maison de Muha

Zakariyā : L'éléphant ! Ô roi du temps!

Le groupe : Il tue les moutons, écrase les poules. La mort apparaît lorsqu'il arrive.

Zakariyyā : L'éléphant ! Ô roi du temps !

Le groupe : À cause de lui les sujets vivent dans l'Insécurité. $\left.{ }^{32}\right\rangle$

On arrive au troisième tableau et on atteint au moment fort tel est l'arrivée face au palais du roi, le sentiment d'anxiété les domine : «Tous les gens se réunissent devant les portes du palais qui occupe une grande partie de la scène ${ }^{33}$. » Pauvre peuple qui poussait des cris de colère sur le grand chemin du palais il ne savait pas combien sa faiblesse et son aveuglement lui devaient coûter cher un jour.

C'est ainsi que les personnages de Wannous montrent au début la voie du palais un déclassement et un dépeuplement d'euxmêmes. Lorsque la foule parvient au palais, la lâcheté la domine et sa conversation manque d'unité. Puis, les voix changent en voix off, elles manifestent leurs émotions anxieuses, leurs cours sont écrasés face à cette puissance supérieure : 
L'espoir de la justice est l'ambition des opprimés dans

«Des voix:

- Mes genoux me lâchent.

- Mon cour bat. (...)

- Je commence à transpirer. (...)

- Ma tête tourne (...)

- Mon cour bat.

- Mon cour bat. ${ }^{34} \gg$

Le peuple prévoit l'autorisation d'entrer dans le château.

Dès le début de ce tableau, on affirme la fascination du palais sur la parole du peuple. Celui-ci est ému par la grandeur de l'espace peu à peu les voix s'affaiblissent, se brisent puis se taisent :

«Cen'est pas par la force que les peuples acceptent les effets d'un gouvernement tyrannique, mais parce qu'ils sont fascinés par un nom, c'est-à-dire par le titre légitime, quel qu'il soit, dont se masque leur souverain, alors qu'il gouverne en tyran. La «façon de régner » tyrannique suppose que le gouvernement dénature les dispositions constitutionnelles du régime, mais cette dénaturation demeure inaperçue, voilée par la permanence du nom ${ }^{35} . »$

Lorsqu'il s'agit de parler de changement, il s'agit de briser la soumission, mais sont-ils au point de rupture du système de pouvoir?, est- il possibles de dépasser ces crises graves ? : « Sur leurs visages se lisent la soumission, la peur et l'embarras. $A u$ fur et à mesure qu'ils avancent dans le palais, ces signes vont être de plus en plus visibles et forts. ${ }^{36}$ »

Les sujets décrivent la cour du roi

Le sentiment d'infériorité domine les sujets, il est évident pour eux que le roi ait en rang de prédominance cela montre que la relation entre le roi et le peuple dépend d rapport de puissance, 


\section{Dr. Iman Mahmoud Zahran}

par ce que le plus fort a toujours eu raison duple se découvre muet face à remarquant dans le palais, où le peć par le château avant que la prédominance du roi, il est fascinc la cour qui révélerait le le roi ait dit un terme. C'est don pouvoir du roi :

«Des voix :

- Est ce que tu vois les jets d'eau?

- Comme dans un rêve.

- Regarde le marbre.

- Il brille de mille feux. [...] jir les tapis.

- Faites attention à ne pas salir léclat du jour. [...]

- Les murs brillent comme l'éclat du jor. [...]

- Nous marchons dans un tunnel labyrinthe. ${ }^{37} \gg$

- Le palais est comme un labyinther a donnant la parole à

Wannous décrit leur façon de marcher, en donnant la parolea Zakariyya :

«La voix de Zakaria : Marchez silencieusement et ne trainez pas les savates. »

Les personnages annoncent leur entrée dans le château :

«-Nous entrons dans le palais. [...]

- Ce hall. [...]

- Nous marchons dans un tunnel en or..»

On remarque ce dialogue ci-dessus décrit l'état d'âme de peuple et sa réaction inattendue lorsqu'il est dans le palais de roi .Il est séduit, sa réplique se change en chuchotements puis en silence : «Des chuchotements étouffés et étonnés se répandent parmi eux..... Un silence pesant. Sans geste ni mouvement. Une masse de corps desséchés et courbés. ${ }^{38} »$ 
L'espoir de la justice est l'ambition des opprimés dans

Le quatrième tableau est intitulé « amãma al-malik » (En face du roi).

La paroisse arrive maintenant au palais qui est, selon les évènements un point central où on découvre le secret et le fondement qui garantit la domination, et la tyrannie. Ce secret n'est que les gardes avec leurs armes et leurs visages dures :

«- À chaque porte ses gardiens.

- La surveillance s'amplifie d'une porte à l'autre. [...]

- Les gardiens sont partout. "39"

Ainsi, le silence, la crainte, et bref, l'étonnement, ont clairement traduit la diminution de la paroisse et la supériorité du roi.

Dans cette pièce, le temps et les actes sont conceptuels. Par ce que les événements historiques se répètent, ce conte peut se passer dans toutes les époques. Aussi même les sujets restent lâches, leurs sensations intérieures sont troublées donc les actions dramatiques de la pièce sont souvent passables pour tout temps :

«Il existe dans tout « fait théâtral » une double temporalité au niveau du temps fictif de la fable. D'une part, les événements se déroulent dans une durée autonome qui peut être considérée, dans certaines pièces, comme une « reproduction mimétique d'une action réelle. ". Et d'autre part, ces mêmes événements évoluent dans une durée réelle, celle de la représentation (une ou deux heures) ${ }^{40} \gg$.

Pourtant la conception politique que la pièce nous évidente: la défaite des sujets face au souverain. Aucun individu n' a de droit humain : 
«Des voix:

- Mes genoux me lâchent.

- Mon cour bat. (...)

- Je commence à transpirer. (...)

- Ma tête tourne (...)

- Mon cour bat.

- Mon cour bat. ${ }^{41}$ "

Malgré cela leur parole expose leur crainte. Cela montre la conception de la pièce : l'écrabouillement du citoyen face à la puissance absolue du roi. Comment réussir enfin une révolution sans quitter cette soumission aveugle?

Puis on doit parler de leur pauvreté, ils ne sont pas dans une très bonne situation économique. La chose la plus étrange est le fait qu'ils sont conscients de leur pauvreté, mais ils ne font rien. Enfin le dernier thème très important concerne la souffrance. Dans les uniques paroles prononcées par eux que le roi ne se laisse pas influencer par leur douleur, laissant ainsi ses sujets dans la négativité leur condition. Ils vivent toujours dans leur routin.

On arrive le tableau final la partie la plus importante s'intitulé Face au roi_: La représentation commune de leur plainte indique une allusion de l'anxiété du roi. Tandis qu'on accuse à peine l'ennemi commun ils cherchent la liberté, et ils se rendent esclaves l'un de l'autre ils veulent l'indépendance et l'unité, et ils se divisent et ils ménagent un tyran Ils sont allés dans des directions différentes, Zakariyyā et le peuple n'étaient pas faits pour le même chemin. La chose la plus incompréhensible est le moment où Zakariyya commence la plainte « L'éléphant ö roi du temps » répète plusieurs fois. Les personnages ne donnent pas d'importance au temps qu'ils ont. Pourtant, ce groupe incapable de présenter sa plainte devant le roi selon l'entrainement de l'initiateur Zakariyya en outre les voix des sujets s'interrompent, ils n'arrivent pas à exposer leur protestation, leurs voix bariolées 
L'espoir de la justice est l'ambition des opprimés dans

au silence : «Face au roi, les voix se défaillent constamment. Quand le roi demande :

Le roi : Qu'est-ce que les sujets veulent de leur roi ?:»

Malgré cela la voix frémissante d'une fillette tente de répondre, mais, immédiatement la voix se dissimuler :

«Une voix : (tremblante qui émane du groupe)

Il a t... (Puis, la voix s'étrangle) ${ }^{42}$ "

Wannous adresse cette question au peuple : comment les sujets se soumettent d'une manière aveugle à un pouvoir contraire à leurs intérêts ? Comment cherchent-ils à l'indépendance alors qu'ils vivent la servitude? Cette servitude aux yeux de Wannous est sans doute un obstacle à la liberté :

Ainsi Louis Janover, rebelle à l'idée de servitude volontaire, écrit :

«Seule la domination est volontaire et son principe ne saurait s'étendre à ceux qui la subissent. ${ }^{43}{ }^{\prime}$

Contrairement à la décision commune les sujets n'osent pas contester le roi et n'arrivent pas à exposer leur plainte devant lui, ils ont un sentiment de perte générale, leur situation reste inchangée, ils tremblent devant roi, ils sont traitres d'euxmêmes. Le chef de groupe Zakariyya est le seul à pouvoir établir une telle communication. Mais Zakariyya n'exprime pas le point de vue du groupe, il effectue un renversement total de la situation à son avantage : tout en s'exprimant au nom du groupe, il déclame une tirade au sujet de «l'attachement «" de la population à l'éléphant. Zakariyya se bat non seulement contre le roi mais aussi contre la faiblesse des gens.

42 lbid.PP.474-475.

43Louis Janover, «La démocratie comme science-fiction de la politique », Réfractions, $\mathrm{N}^{\circ} 12$, Printemps 2004, pp. 92-93. 
après l'échec du peuple, traduit cette chute avec un inversement absolu dans un long discours comme situation, en déclarant au roi peuple venait pour_affirmer une représentant et leader de ce peúléphant- cette lâcheté augmente demande générale de marier l'éló du «nous » dans la réplique leurs malheurs au futur. L'emp' il est le délégué de groupe : de Zakariyya confirme au roi qu'

«Zakariyyā : Nous, nous aimons l'éléphant, Ô roi du temps !...] Mais nous avons remarqué que l'éléphant est toujours seul. [...] C'est pourquoi nous avons pensé à venir, nous les sujets, pour demander que l'éléphant soit marié afin diminuer sa solitude. Ainsi, il nous donnera des milliers d'éléphants. d'éléphants, des centaines d'éléphants, d'éléphants. ${ }^{44}$ "Le peuple De la sorte, la ville sera remplie d'élép le peuple, bon et crédule, bon et crédule n'a pas connu la lib jamais haï le roi. Le peuple ne parce qu'il est sans ambition, n'a Jam'un piège qui protège la se soulève pas. L'éléphant n'est qu'un piège qui protège la force contre le peuple.

L'écroulement final est inattendu puisque le roi a nommé Zakariyya accompagnateur de l'éléphant. Ainsi, Zakariyya se sépare certainement_du groupe. Le peuple a mal orienté sa volonté il sort du palais pour préparer un mariage convenable de l'éléphant :

«Les hommes, sous l'emprise de la servitude volontaire, sont susceptibles de surmonter la peur de la mort, d'accueillir la destruction de soi au point d'offrir leur vie au tyran ou à celui qui occupe le lieu du pouvoir ${ }^{45}$. $)$ 
L'espoir de la justice est l'ambition des opprimés dans

De la pérennité de la tyrannie comme modèle de domination:

La pièce de Wannous applique la théorie de la servitude volontaire " d'Etienne de La Boétie ${ }^{46}$ "et tout au long de la pièce notre dramaturge pose cette interrogation habile et essentielle : " pourquoi les individus obéissent-ils à leurs gouvernants? ${ }^{47} \gg$

La thèse de la Boétie est la suivante : " les régimes sont fondés sur la peur, laquelle sert à dissimuler l'absence de légitimité des gouvernants. Ainsi, le peuple $s$ 'auto-soumet aux pouvoirs en place, par simple habitude, par récurrence historique. " Ce qui fait agir les individus en société, c'est précisément de se penser comme, individu libre et responsable, ayant une tâche à accomplir :

«Le terme tyran a pris un sens péjoratif pour désigner un individu qui détient tout le pouvoir et l'exerce en faisant régner la terreur de manière despotique, injuste, cruelle, sans respect la $\left.101^{48}\right)$

\section{L'asservissement est volontaire.}

Malgré que ce roi soit la cause de leurs pauvreté, comment les sujets obéissent-ils au roi, $n$ 'a pas de pouvoir que celui ses sujets confient? «Ainsi, puisque le tyran ne tire sa puissance que du peuple, il suffit de « ne plus le soutenir » pour que cette puissance s'écroule d'elle-même49 »Il a fait d'eux des marionnettes sans têtes amorphes, des choses sourdes et muettes, Wannous accuse les lâches qui courbent les

46 Note sur Le Discours de la servitude volontaire est une cuvre d'Etienne de La Boétie, dont l'influence sur la philosophie politique est très grande.) Le Discours de la servitude volontaire ou le Contr'un est un ouvrage rédigé en 1547[réf. nécessaire] par Étienne de La Boétie à l'âge de dix-huit ans. Sa première publication date de 1576 .

Ce texte consiste en un court réquisitoire contre l'absolutisme qui étonne par son érudition et par sa profondeur, alors qu'il a été rédigé par un jeune homme d'à peine dix-huit ans. Ce texte pose la question de la légitimité de toute autorité sur une population et essaie d'analyser les raisons de la soumission de celle-ci (rapport " domination-servitude »)

https://fr.wikipedia.org/wiki/Discours_de_la servitude_volontaire.)

48 La Boétie Dolontaire Mercredi 20 jüin $20 \overline{07}$, par Rodophe Gouin

http_WWW.dokars de la servitude volontaire, analyse, 3 août 2012 par Lionel Hottin

49 Aimer-la-littéra.nc-la-Boétie-de-la-servitude- volontaire-analyse.

Renaissance par cotenting Boétie, Discours de la servitude volontaire, 1576 Posté dans Essai, La

asistaine 
dos par peur du roi pou
souhaitent possible.

Dr. Iman Mahmoud Zahtian pour acquérir la sécurité perdue qu'ils

Le premier reproche de Wannous en tant qu'il est un foyer actif montre son point de vue contre ce peuple "insensé », c'est-à. dire impuissant d'améliorer sa propre situation.

De plus, Wannous attaque avec vigueur la représentation négative et l'indifférence du peuple face au malheur qu'il subit et qui se satisfait à son propre esclavage : "opiniâtres à leur mal ». Les gens sont aveugles, ils n'ont jamais atteint la lumière de la liberté.

On note, par exemple que Wannous recourt à une forme d'ironie pour mettre en valeur le reproche. Il confirme la soumission du gens : "Vous vous affaiblissez afin qu'il soit plus fort ${ }^{50}$ ». Il faut bien indiquer que ce n'est pas au roi mais au peuple que s'attaque Wannous.

De même Wannous, rappelle au peuple sa responsabilité de culpabilité : c'est lui qui a permis au roi d'atteindre son autoritaire : " celui-là même que vous avez fait ce qu'il est ». Le peuple a donc fabriqué lui-même son propre « ennemi », Néanmoins c'est le peuple, principalement, qui lui permet de s'y imposer en étant son serviteur : « les moyens que vous lui fournissez pour vous détruire ${ }^{51} \gg$.

Le roi force et oblige la foule à obéir et à reconnaitre qu'elle est misérable est parce qu'elle s'habitue à la servitude et oublie son droit d'être libre :

«La première raison pour laquelle les hommes servent volontiers, est parce qu'ils naissent serfs et sont nourris comme tels. ${ }^{52}$ »

50 Ibid.

51 Thomas More, Livre 1,Aimer-la-littérature La Boétie, Discours de la servitude volontaire, 1576 Posté dans Essai, La Renaissance par cotentinghislaine

52La Boétie, Discours de la servitude volontaire, analyse, 3 août 2012 par Lionel Hottin http WWW.dokamo.nc-la-Boétie-de-la-servitude- volontaire-analyse 
L'espoir de la justice est l'ambition des opprimés dans

Il ne faut donc pas négliger que, Wannous fait du sujet le concept central de la pièce. De même Wannous porte son de La Boétie: comment peut-il se faire que Il confirme la question de bourgs, tant de villes, tant de nations " tant d'hommes, tant tyran seul, qui n'a de puissance que celle qu'ent quelquefois un (turan seuls qui n'a dent? ".

Wannous affirme que la société est divisée entre ceux qui s'habitue de vivre dans une société peuple oublie sa liberté, et la faiblesse

Hegel écrit : "Les monarques ne se distinguent pas des autres hommes par la force physique ou par leurs qualités d'esprit, et pourtant, des millions d'hommes acceptent d'être soumis à leur
autorité. C'est une soumission acceptée : «La servitude volontaire n'est donc qu'un type d'obéissance particulièrement difficile à comprendre, en ceci qu'elle est complicité de notre propre état de privation de liberté. », Comme l'écrit S. Weil : "c'est quand sévit la mort que le miracle de l'obéissance éclate aux yeux ${ }^{54} "$

Il y a des raisons pour lesquelles les sujets servent habituellement, premièrement, ces gens sont toujours habitués à une obéissance aveugle, une soumission entière aux ordres de roi.

La Boétie décrit dans son "Discours »:

«Les hommes nés sous le joug, puis nourris et élevés dans la servitude, sans regarder plus avant, se contentent de vivre comme ils sont nés et ne pensent point avoir d'autres biens ni d'autres droits que ceux qu'ils ont trouvés ; ils prennent pour leur état de nature l'état de leur naissance ".

Deuxièmement, c'est que devant la puissance outrée de roi les sujets sont : « lâches et efféminés ». Il reste encore à déterminer

53G.W.F. Hegel, Principes de la philosophie du droit, texte présenté, traduit et annoté par R.

Derathé,Vrin, Paris, 1975, p. 296.

54Weil, $1978: 88$ ). WEIL, S., « Méditation sur l'obéissance et la liberté ", in LA BOÉTIE É. (DE), 1978. 
positivement ce rapport, duquel Wannous tente néanmoins
d'affirmer que la difficulté commence lorsque, les sujets sont
coutumiers l'assujettissement coutumiers l'assujettissement donc, la liberté n'a évidemment pour ces lâches aucune valeur. Ce type de sujets ne lutte plus pour protester: "N'oublie pas c'est l'éléphant de roi " $\mathrm{Au}$ contraire, le roi les force à se soumettre :

«Les tyrans ne sont grands que parce que nous sommes à genoux. " (Discours de la servitude volontaire)

De l'aveuglement des peuples notre dramaturge voit que le peuple se laisse dépouiller sans résister, il se laisse tromper volontiers :

"Pour que les hommes, tant qu 'ils sont des hommes, se laissent assujettir, il faut de deux choses l'une : ou qu'ils y soient contraints, ou qu'ils soient trompés. " (Discours de la servitude volontaire)

Sans aucun doute l'habitude explique la domination, pour La Boétie, l'habitude c'est l'oubli de la liberté qui est, à l'origine, entretenu par la ruse du tyran. Pour L. Althusser :

" La ruse du tyran vaut bien comme cause de la servitude volontaire, mais dans un monde où règne nécessairement l'habitude. ${ }^{55}$ "

\section{La liberté lâchée}

La question de la domination acceptée est alors question de la totalité d'une formation sociale.

L'important est de noter que Wannous, ayant fait de cette question le cœur de la pièce, en outre, il met en lumière la cause de la légitimité des puissants de dominer le peuple. En supportant une vision sur la relation le roi et les sujets, Wannous aborde tout au long de la pièce une affirmation principale telle que : le pouvoir du roi se fonde uniquement sur l'acceptation commune de peuple. Lorsque le peuple repousse cette force, la puissance du dictateur s'effondre : « Le pouvoir des tyrans ne repose que sur l'abandon du pouvoir du peuple. ...... Le tyran

55 L. Althusser, La Transformation de la philosophie : conférence de Grenade (1976) 
L'espoir de la justice est l'ambition des opprimés dans est souvent un homme faible, comme les autres. Seuls les
crédules peuvent l'idolâtrer. ${ }^{56}$ "

Le lien entre dominants et dominés:

Il faut préciser quelques points, tout d'abord le coup de génie de Wannous est d'avoir montré dans la pièce que le peuple ne se rebelle pas contre la tyrannie de roi. Selon cette perspective le peuple loin d'être des sujets passifs qui se laissent accepter d'être trompés :

«Avec La Boétie : les dominés ne seraient pas trompés par leurs maîtres, mais s'auto-tromperaient en quelque sorte pour finir par s'autodétruire ")

Puisque comme on vient de souligner, le peuple par son inactivité, est aussi coupable de sa servitude. Le peuple doit éliminer la cause ce cette servitude. Pour quitter cette attitude, il exige un certain courage mais aussi une connaissance. J. P. Marat affirme que :

«Bien au contraire, il ne cesse d'affirmer que la clef de la liberté est entre les mains du peuple ${ }^{57} \gg$.

Les sujets manquent de courage pour quitter leur état de soumission la plus entière et l'obéissance la plus aveugle :

Marat reproche la négativité des peuples: «Le peuple ne se laisse pas seulement enchaîner : il présente lui-même sa tête au joug...Non content d'être la dupe des fripons, le peuple va presque toujours au-devant de la servitude et forge lui-même ses fers ${ }^{58}$.»

La pièce de Wannous a vivement confirmé que la servitude du peuple est un appel interne. C'est pourquoi, il appuie que :

«Beaucoup s 'imaginent que la servitude est forcée, alors qu'elle est toute volontaire ${ }^{59} »$

56 La Boétie, Discours de la servitude volontaire, analyse, 3 août 2012 par Lionel Hottin http_WWW.dokamo.nc-la-Boétie-de-la-servitude-volontaire-analyse

57 J. P. Marat, Les Chaînes de l'esclavage, 10/18, Paris, 1972, p. 39 et p. 247.

58 Ibid.

59 P. CLASTRES, Liberté, malencontre, innommable, in édition du Discours de la servitude volontaire, Payot, Paris, 1976. p. 110-111. 


\section{Dr. Iman Mahmoud Zahran}

\section{CONCLUSION}

À ce point, on pourrait montrer que cette pièce qui a eu un très grand succès, repose sur cette conception essentielle, le peuple se laisse ruiner lui-même par sa corruption de soutenir et flatter l'ordre corrompu de roi.

On pourrait montrer que Wannous a vu que cette servitude volontaire est une maladie inguérissable, et que la pratique du régime despotique bloque le chemin de l'humanité :

"Les mains des lettrés sont pures, mais ils n'ont pas de mains.. Contre la servitude volontaire des peuples, aucun moyen solide ne vaut. ${ }^{60} \gg$

Saad Allah Wannous a la capacité de prévoir les événements, on peut affirmer que l'indépendance des peuples arabes de la servitude volontaire est sa préoccupation primordia celle-ci est l'arme montrant l'importance de la voix coherente ce sur le trajet entre le des peuples écrasés cette arme: "L'hom pas seulement un début de la formation jusqu'à la fin n est pinsi, les signés de

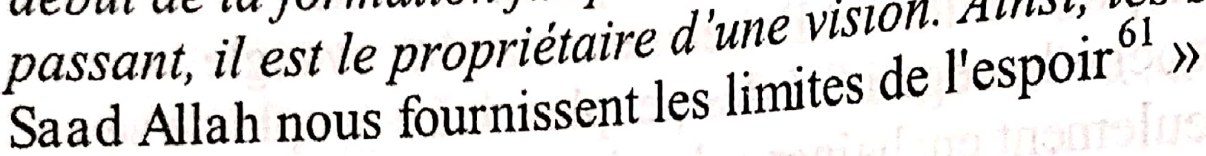

Il ne fait aucun doute que le plupart des peuples arabes ont brisé le silence et la peur et ont prouvé dans leur révolte montre un courage inégalé .Toutefois, la catégorie dont ils sont mentionnés dans le jeu, ils sont encore loin de la révolution sous le prétexte de la peur et de la lâcheté. Certains d'entre eux croient même dans la révolution une vertu chaotique qui remonte au Moyen Age.

Pour ces silencieuse on dit : votre silence remplacera des nouvelles générations d'éléphants les plus ridicules et les plus

60 Emmanuel Buron, «La nature et ses façons : l'anthropologie politique humaniste d'Etienne de La Boétie », Fabula / Les colloques, Étienne de La Boétie, Discours de la servitude volontaire, URL : http:/www.fabula.org/colloques le 20 janvier 2015.

61 Aziz Hilal, «Réappropriation du passé : le théâtre arabe face aux textes médiévaux », Babel, $15 \mid 2007,87-126$. 
L'espoir de la justice est l'ambition des opprimés dans

injustes de ses prédécesseurs et vous vous serez les premières victimes.

C'est l'occasion pour vous d'avoir terminé votre silence et pour rejoindre la révolution et être au niveau des sacrifices et les gens ont connu les raisons de la vie inévitable.

Dans la pièce «Éléphant, ô roi du temps » Wannous se maintient sur une histoire diffusée dans le monde arabe, il la divise en tableaux, et sans indiquer des noms aux personnages. À la fin de de la pièce les représentants se lèvent pour confirmer aux spectateurs que c'était une histoire, et ils sont des représentants ils les apprennent que lorsque les éléphants se diffusent les gens deviennent lâches et, ne peuvent pas exiger leurs droits - comme indiqué l'histoire.

De tout ce qui précède on peut affirmer que notre dramaturge est le créateur du théâtre politique en Syrie, par lequel il personnifie le rapport injuste entre le citoyen et le pouvoir.

A travers cette pièce on se demande si Wannous a réussi à mener ses pensées pour les peuples arabes? La réponse et que notre dramaturge a parvenu de fonder par ce plateau la conscience des nations arabes à travers la création du théâtre politique.

La pièce est terminée par la prévision suivante :

«Tout le monde: C'est un conte,

Acteur 5 : Nous sommes des Représentants

Acteur 3: nous le jouons pour vous apprendre la leçon.

Acteur 3 : Nous vous l'avons jouée (l'histoire de l'éléphant) pour en tirer avec vous une leçon.

Acteur 7 : Est-ce que vous savez maintenant pourquoi les éléphants existent?

Actrice 3 : Est-ce que vous savez maintenant pourquoi les éléphants prolifêrent?

Acteur 7 : Saviez-vous maintenant pourquoi se multiplient-ils les éléphants ?

Acteur 4: Quand les éléphants commencent multiplient c'est une autre histoire. 
Tout le monde : une histoire violente et sanglante. ${ }^{62} "$

Voici la prévision a été effectuée et les éléphants augmentent et le sang et la cruauté s'accroissent parce que la durée de ces éléphants devient insupportable, le peuple se rebelle contre l'éléphant et son conservateur. Malgré le fait que notre grand dramaturge mourut, après une longue et courageuse lutte contre le cancer, en 1997, son théâtre reste la source importante pour le cancer, en 1997, son théâtre reste la soure l'autorité et les peuples
concevoir la relation déséquilibrée entre labes.

Wannous n'est pas comme les autres dramaturges de son temps, malgré les défaites successives qu'il a vécues, seul l'espoir qui a nourri son désir.

Cependant, le drame s'est terminé par une prophétie, la vie sera meilleure pour les gens quand ils parleront, lorsqu'ils se débarrassent de l'esclavage du silence. Zakariyya a essayé d'éveiller leur capacité de parler, mais la crainte qui les domine la font échouer au premier test et ne peuvent pas se défendre. Saad Allah dit que la prolifération des éléphants début d'une autre histoire. «Un conte violent et sanglant »La prolifération des éléphants se porte dans un solide les raisons de leurs fins inévitables qui sera sanglante, non seulement pour l'éléphant mais aussi pour ses esclaves.

On voit comment les éléphants sont tombés par les peuples arabes de Tunisie, l'Egypte, la Libye et ils continuent à lutter en Syrie aujourd'hui, la patrie de Wannous. Les mots Saadallah restent dans nos oreilles comme il se prononçait hier. Ces mots résument la culture d'un sage venu avant le temps et parti avant la date du départ. Dieu bénisse Saadallah, et à aider les peuples arabes de faire tomber les autres éléphants tyrans. Il est le premier grand intellectuel qui se considère comme une référence pour les générations à venir. 
L'espoir de la justice est l'ambition des opprimés dans

\section{Corpus de Wannous :}

\section{Bibliographie}

L'éléphant ô roi du temps ! Traduit en français par, Batoul Jalabi-Wellnitz, Publications de l'Institut français du ProcheOrient

Chapitre I. al-fīl yā Malik Al-Zamān (L'éléphant Ô roi du Temps!)

Collection : Études arabes, médiévales et modernes | PIFD 225

Année d'édition : 2006.

\section{Ouvrages critiques sur S. Wannous:}

La revue 'Adab wa naqd, Damas, n 143, juillet 1997, p. 98.

La revue al-Ma'rifa n 68, avril 1969.

Hilal Aziz, « Réappropriation du passé : le théâtre arabe face aux textes médiévaux », Babel, 15 | 2007.

Nada TOMICHE traduit ce terme par « non réalisme ».

Cette formulation est empruntée à Erwin Piscator, Le théâtre politique,

TOMICHE, Nada, La littérature arabe contemporaine roman, nouvelle, théâtre. Maisonneuve Edi. Larousse, Paris, 1981.

Samarkand Mohammed Habib, Le théâtre arabe au miroir de lui-même et son contact avec les créations des deux rives de la Méditerranée Presses Uni:du Mirail, 2008, Numéro 58 de Horizons maghrébins

Stéphane Capron Journalist au service culture http://www.franceinter.fr/dossier-le-theatre-humaniste-dewannous .

TOMICHE, Nada, La littérature arabe contemporaine roman, nouvelle, théâtre. Maisonneuve Edi. Larousse, Paris, 1981, p. 131

WANNOUS, Saadallah. Euvres complètes, Al-a'mal alkāmila (Euvres complètes), , Damas, al- Ahālī, 1996, tome 3. 


\section{ouvrages sur la servitude volontaire de La Boétie :}

Www.Fabula.org/colloques/documen2490.php.consultée le 5-52015

ABENSOUR, M. et GAUCHET, M., 1978. «Présentation. Les leçons de la servitude et leur destin », in LA BOÉTIE, É. (DE), 1978.

ALTHUSSER, L., 1976. «Idéologies et appareils idéologiques d'État », Positions, Paris : éd. Sociales.

Althusser, L. La Transformation de la philosophie : conférence de Grenade (1976).

ARENDT, H., 1990 (1951). Le système totalitaire, Paris : Seuil.

Boireau-Rouillé Monique,Réfractions n 17 , hiver 2006, Pouvoir et conflictualités Conflictualité et politique

BOURDIEU, P., 1989. La Noblesse d'État: Grandes

écoles et esprit de corps, Paris : Minuit.

BOURDIEU, P., 1997. Les méditations pascaliennes,

Paris : Seuil.

BOURDIEU, P., 1992. Réponses, Paris : Seuil.

Buron Emmanuel, « La nature et ses façons :

l'anthropologie politique humaniste d'Etienne de La

Boétie », Fabula / Les colloques, Étienne de La

Boétie, Discours de la servitude volontaire, URL :

http://www.fabula.org/colloques/document2490.php,

Article publié

le 20 janvier 2015.

CLASTRES, P., 1978. «Liberté, Malencontre, Innommable », in LA BOÉTIE, É. (DE), 1978.

FOUCAULT, M., 2001. «Le sujet et le pouvoir », Dits et écrits II, 1976-1988, Paris : Gallimard.

G.W.F. Hegel, Principes de la philosophie du droit, texte présenté, traduit et annoté par R. Derathé,Vrin, Paris, 1975. 
L'espoir de la justice eat l'ambition des opprimbs dan

Gouin, Rodolphe (2007), «e Servitude volontaire ${ }_{3}$ in V. Bourdeau et R. Metrill (dir.), Dicopo, Dictionnaire de théoric
politique.

Hottin Lionel , La Bodte, Discours de la servitude volontaire, analyse, 3 aout 2012 htip WWW dokamo nc-la-Botic-de-laservitude-volontaire-analyse

Janover Louis, eLa démocratic comme science-fiction de la politique ", Réfractions, No 12, Printemps 2004

J.M. Rey, La part de l'autre, P.U.F., Paris, 1998.

J. P. Marat, Les Chaines de l'esclavage, 10/18, Paris, 1972.

LA BOETIE, E. (DE), 1978 (1576). L'Ecosse Des Clans et Des

Tartans

MUNRO R. W., DURING Thérèse ( Texte Français)

Published by Les Éditions La Boćtie, Paris, 197

Le discours de la servitude volontaire, Paris : Payot. 17 novembre 2002 LEFORT, C., 1978. «Le nom d'Un », in LA BOÉTIE É. (DE),
1978.

1. MARX, K. et ENGELS, F., 1974 (1845; texte publié en 1932). L'idéologie allemande, Paris : éditions sociales.

MARX, K. et ENGELS, F., 1998 (1848). Manifeste du Parti communiste, COLLECTIF, Le Manifeste communiste aujourd'hui, Paris : Les Éditions de l'Atelier/Éd. Ouvrières.

MARX, K., 1997 (1851). Le 18 brumaire de Louis Bonaparte, Paris : éd. Mille et une nuits

More Thomas Utopie, Livre I, Aimer-la-littérature La Boétie, Discours de la servitude volontaire, 1576 Posté dans Essai, La Renaissance par cotentinghislaine.

R. Bodei, La Géométrie des passions, P.U.F.,

Paris, 1967.

Spinoza, le Court Traité CEuvres Complètes, La Pléiade, Gallimard, Paris, 1954.

UBERSFELD, Anne, Lire le théâtre, tome 1, BELIN ,1996. WEBER, M., 1995 (1971). Économie et société, Paris : Plon, rééd. Agora.

WEIL, S., " Méditation sur l'obéissance et la liberté », in LA BOÉTIE É. (DE), 1978. 